\title{
Directed transdifferentiation of Müller glial cells to photoreceptors using the sonic hedgehog signaling pathway agonist purmorphamine
}

\author{
DANDAN GU ${ }^{1,2}$, SONGTAO WANG ${ }^{2}$, SHUAI ZHANG ${ }^{3}$, PENG ZHANG $^{2}$ and GUOMIN ZHOU ${ }^{1,3}$ \\ Departments of ${ }^{1}$ Anatomy, Histology and Embryology, and ${ }^{2}$ Integrative Medicine and Neurobiology, \\ Institute of Acupuncture Research, School of Basic Medical Sciences; ${ }^{3}$ Key Laboratory of Medical Imaging Computing and \\ Computer Assisted Intervention of Shanghai, Fudan University, Shanghai 200032, P.R. China
}

Received November 30, 2016; Accepted July 28, 2017

DOI: $10.3892 / \mathrm{mmr} .2017 .7652$

\begin{abstract}
Specification of distinct cell types from Müller glial cells is key to the potential application of endogenous repair in retinal regeneration. Sonic hedgehog $(\mathrm{SHH})$ has been established as a potent mitogen for rat Müller glial cells, which also induces Müller glial cells to dedifferentiate and adopt the phenotype of rod photoreceptors. The present study investigated the effects of purmorphamine, a small molecule that activates the SHH-pathway, in the proliferation, dedifferentiation and transdifferentiation of Müller glial cells, as determined by several methods including immunofluorescence, polymerase chain reaction and western blotting. It was demonstrated that it may be able to replace $\mathrm{SHH}$ for the regeneration of retinal neurons. Purmorphamine was revealed to stimulate the proliferation of Müller glial cells by increasing the expression of cyclin D1 and cyclin D3. In addition, purmorphamine-treated Müller glial cells were induced to dedifferentiate by inducing the expression of progenitor-specific markers; subsequently differentiating into rod-like photoreceptors. Intraocular injection of purmorphamine promoted the activation of Müller glial cells, and in turn, the production of rod-like photoreceptors in acute damaged retina. These results suggested that the endogenous neurogenic capacity of retinal Müller glial cells may be enhanced by this small molecular agonist of the $\mathrm{SHH}$ signaling pathway.
\end{abstract}

Correspondence to: Dr Dandan Gu or Dr Guomin Zhou, Department of Anatomy, Histology and Embryology, School of Basic Medical Sciences, Fudan University, 131 Dongan Road, Shanghai 200032, P.R. China

E-mail:dd.g@163.com

E-mail: gmzhou@shmu.edu.cn

Key words: Müller glial cell, purmorphamine, sonic hedgehog, rod cell, retinal regeneration

\section{Introduction}

Injuries and diseases that induce retinal neuronal cell death may result in permanent blindness. Stimulating endogenous neuronal regeneration is a potential strategy to treat these retinal lesions. In non-mammalian vertebrates, endogenous repair to restore neurons occurs very efficiently, even following complete loss of the retina, either through retinal pigment epithelium transdifferentiation or through stem cell proliferation and differentiation (1-4). However, these endogenous repair processes in mammalian retina are limited $(5,6)$, as the mammalian retina have no significant persistent neurogenic source such as ciliary marginal zone; in mice, neurogenesis is completed within the first 10 postnatal days (7). Nevertheless, great efforts have been made in the past few decades to overcome the limits of these regenerative processes in mammalian retina $(1,8-11)$.

The field of retinal regeneration from Müller glia-derived progenitor cells in mammals has recently become a promising area of interest $(1,2,12)$. Müller glia are the sole glial cells generated by multipotential retinal progenitors. In several vertebrate classes, in response to acute retinal injury, Müller glial cells are able to dedifferentiate, proliferate and acquire a progenitor-like state $(1,8,9)$. In the retina of zebrafish, it has been reported that when retinal neurons are destroyed, appropriate replacements for these missing retinal cells are produced via progenitor cells derived from Müller glia $(10,11)$. A previous study demonstrated that functional regeneration of the zebra fish retina is also achieved through progenitors derived from Müller glia (11). These progenitor cells cycle rapidly, progressing through the radial process of daughter Müller glial cells to reach the outer nuclear layer (ONL), where they exit the cell cycle and begin to differentiate into rod photoreceptors. In N-methyl-D-aspartate-treated chicken retina, Müller glial cells in the central retina re-enter the cell cycle, dedifferentiate, acquire progenitor-like phenotypes and produce new neurons (1). Our previous studies have revealed that acute retinal degeneration induced by a N-methyl-N-nitrosourea (MNU)-injection promoted the transdifferentiation of Müller glial cells into photoreceptors $(13,14)$. The results from these previous studies suggested that Müller glial cells may serve as a potential source of neural regeneration in the mammalian 
retina; however, the regenerative ability varies greatly across different species, with warm-blooded species exhibiting the lowest regenerative ability $(1,3,8-10)$. Therefore, a number of different methodologies have been evaluated for the regulation of the endogenous repair capacity of retinal Müller glial cells in warm-blooded vertebrates, amongst which the sonic hedgehog ( $\mathrm{SHH})$ signaling pathway is considered one of the most important (13,15-18).

A previous study involving zebrafish suggested that SHH signaling is required for the proliferation and survival of retinal progenitor cells (19). Similarly, retinal marginal progenitors also depend on $\mathrm{SHH}$ as a mitogen in chicks and in mice $(7,20,21)$. Our previous study also revealed that $\mathrm{SHH}$ was crucially involved in stimulating the proliferation of Müller glial cells in vitro, and exogenous $\mathrm{SHH}$ exposure exerted proliferative effects on Müller glial cells in vivo following retinal injury (14). In addition, SHH-treated cells were shifted to neural lineage by expressing neuron-specific class III $\beta$-tubulin (Tuj1), directing cell fate to rod cells (14).

Although the activity of a commercially available $\mathrm{SHH}$ was improved through a mutation at the amino (N)-terminus, as a protein, the activity remains variable. Purmorphamine is a small molecule that activates $\mathrm{SHH}$ signaling, potentially through Smoothened (22). Therefore, the present study investigated whether SHH may be replaced by purmorphamine in the transdifferentiation of Müller glial cells to retinal neurons, and thus, attempted to provide a more convenient, effective and stabilized therapy.

\section{Materials and methods}

Ethical statement. The present study was approved by the Ethics Committee of Fudan University (Shanghai, China). The protocol involving the use of animals adhered to Statement for the Use of Animals published by the Association for Research in Vision and Ophthalmology (23), and the experiments were conducted in accordance with Shanghai Experimental Aanimal Management Method and Fudan University Guide for the Care and Use of Laboratory Animals $(24,25)$.

Müller glial cell culture. Primary cultures of retinal Müller glial cells were prepared as previously described (14). Briefly, the eyes from postnatal day 7 Sprague-Dawley rats (5 rats each time, male, weighing $20 \mathrm{~g}$, supplied by Department of Laboratory Animal Science of Fudan University) were enucleated under sterile conditions. The retinal tissues were then digested in $0.25 \%$ trypsin and $0.1 \%$ type I collagenase at $37^{\circ} \mathrm{C}$ for $5 \mathrm{~min}$. Dissociated retinal cells were plated onto tissue culture dishes in monolayer-culture medium, which was composed of Dulbecco's modified Eagle's medium/F12 supplemented with N-2 Supplement, $2 \mathrm{mM}$ glutamine, $0.1 \%$ penicillin-streptomycin and $10 \%$ fetal bovine serum (all purchased from Invitrogen; Thermo Fisher Scientific, Inc., Waltham, MA, USA), and the plates were incubated at $37^{\circ} \mathrm{C}$ in humidified atmosphere containing $5 \% \mathrm{CO}_{2}$. The culture medium was changed every 2 days. A further purified flat cell population was obtained after 3 passages.

Cell transdifferentiation methodology. To examine the regenerative potential of Müller glial cells, $1 \times 10^{4}$ cells $/ \mathrm{ml}$ were plated on poly-D-lysine $(500 \mu \mathrm{g} / \mathrm{ml})$ and laminin $(5 \mu \mathrm{g} / \mathrm{ml})$ coated glass coverslips. To measure the effects on proliferation, the $20 \mathrm{kDa}$ N-terminal signaling domain of SHH (SHH-N; 10 or 20 nM; R\&D Systems, Inc., Minneapolis, MN, USA) and purmorphamine $(0.1$ or $0.5 \mu \mathrm{M}$; Sigma-Aldrich, Merck KGaA, Darmstadt, Germany) were added to the culture medium, with or without cyclopamine (10 $\mu \mathrm{g} / \mathrm{ml}$; Sigma-Aldrich, Merck $\mathrm{KGaA}$ ) on the first day of culture and maintained at the same concentration throughout the 2-day culture period. A total of 7 treatment groups were established: i) $10 \mathrm{nM} \mathrm{SHH}-\mathrm{N}$; ii) $20 \mathrm{nM}$ SHH-N; iii) $0.1 \mu \mathrm{M}$ purmorphamine; iv) $0.5 \mu \mathrm{M}$ purmorphamine; v) $20 \mathrm{nM}$ SHH-N $+10 \mu \mathrm{g} / \mathrm{ml}$ cyclopamine; vi) $0.5 \mu \mathrm{M}$ purmorphamine $+10 \mu \mathrm{g} / \mathrm{ml}$ cyclopamine; and vii) the control group (culture medium only). In addition, Dickkopf-related 1 (DKK1, $0.1 \mu \mathrm{g} / \mathrm{ml}$; R\&D Systems, Inc.) was added to purmorphamine-stimulated Müller glial cells to determine whether the Wnt pathway was involved. Following 2 days of culture, cells on the coverslips were fixed in $4 \%$ paraformaldehyde at $4^{\circ} \mathrm{C}$ for $10 \mathrm{~min}$ and processed for immunocytochemistry to detect proliferation-associated markers. Progenitor cell markers were evaluated following 7 days of treatment with purmorphamine or SHH-N. Cell proliferation was examined by adding 5-bromo-2'-deoxyuridine (BrdU, $10 \mu \mathrm{M}$; R\&D Systems, Inc.) to the culture medium during the final $18 \mathrm{~h}$ of this 7-day treatment. Subsequently, the cells were transferred to fresh culture medium, without purmorphamine or SHH-N, for a further 2 days to investigate Müller glia-derived cell differentiation.

Intravitreal injection. Photoreceptor apoptosis was induced in Sprague Dawley ${ }^{\circledR}$ rats (male, aged 8-10 weeks, 300 g, 7 rats per group, repeated 3 times, supplied by Department of Laboratory Animal Science of Fudan University) by a single intraperitoneal injections of $60 \mathrm{mg} / \mathrm{kg}$ MNU (Sigma-Aldrich, Merck KGaA). All the animals were kept in an air-conditioned room at $22 \pm 2^{\circ} \mathrm{C}$ and $60 \pm 10 \%$ relative humidity under a $12: 12 \mathrm{~h}$ light/dark cycle (lights on at $7 \mathrm{am}$ ), food and water were available ad libitum. The animals were treated according to the Fudan University Guide for the Care and Use of Laboratory Animals.

Immediately following MNU administration, the left eyes (control group) were intravitreally injected with sterile saline and the right eyes (treatment group) were injected with purmorphamine (260 ng) or SHH-N (400 ng), with or without cyclopamine $(5,000 \mathrm{ng})$; all injection volumes were $5 \mu \mathrm{l}$ and the rats received daily injections for 7 consecutive days. Rats were then sacrificed with $10 \%$ chloral hydrate on day $1,3,7$ or 15 . Enucleated eyes were harvested and fixed in $4 \%$ paraformaldehyde at $4^{\circ} \mathrm{C}$ overnight. The cryostat section $(10 \mu \mathrm{m})$ were produced as previously described (13).

Immunofluorescence analysis. Immunofluorescence was performed as previously described (13) using the following primary antibodies: Mouse anti-proliferating cell nuclear antigen (cat. no. 2586S; PCNA; 1:1,000; $4^{\circ} \mathrm{C}$ overnight; Cell Signaling Technology, Inc., Danvers, MA, USA); rabbit anti-glutamine synthetase (cat. no. G2781; GS; $1: 2,000 ; 4^{\circ} \mathrm{C}$ overnight; Sigma-Aldrich, Merck KGaA); mouse anti-vimentin (cat. no. V6630; 1:20; $4^{\circ} \mathrm{C}$ for 48 h; Sigma-Aldrich, Merck $\mathrm{KGaA}$ ); rabbit anti-vimentin (cat. no. $2707-1 ; 1: 200 ; 4^{\circ} \mathrm{C}$ overnight; Epitomics, Inc.; Abcam, Cambridge, MA, USA); mouse 
Table I. Polymerase chain reaction primer sequences.

\begin{tabular}{|c|c|c|}
\hline Gene & Accession no. & Sequence $\left(5^{\prime} \rightarrow 3^{\prime}\right)$ \\
\hline \multirow[t]{2}{*}{ GAPDH } & NM_017008 & F: CTGCCCAGAACATCATCCCT \\
\hline & & R: TGAAGTCGCAGGAGACAACC \\
\hline \multirow[t]{2}{*}{$n r l$} & NM_001106036 & F: GCCTGAGGTCCCTGGAATGAGTGT \\
\hline & & R: TAGTGTTTGGGGCGGGGAAGAT \\
\hline \multirow[t]{2}{*}{$\operatorname{crx}$} & NM_021855 & F: CCTCACTATTCGGTCAATGCC \\
\hline & & R: ATGTGCCTGCCTTCCTCTTC \\
\hline \multirow[t]{2}{*}{ smo } & NM_012807 & F: CACCTCCAGCGAGACCCTA \\
\hline & & R: AGCCTCCCACAATAAGCA \\
\hline \multirow[t]{2}{*}{ glil } & NM_001191910 & F: GTGGCAACAGGACGGAACTT \\
\hline & & R: CGACTGTGAGACCCTATACCC \\
\hline
\end{tabular}

Crx , cone-rod homeobox; F, forward; $n r l$, neural retina-specific leucine zipper; gli1, GLI family zinc finger 1; R, reverse; smo, smoothened homolog precursor.

anti-nestin (cat. no. MAB353B; 1:500; $4^{\circ} \mathrm{C}$ overnight; EMD Millipore, Billerica, MA, USA); mouse anti-paired box protein 6 (cat. no. AB 528427; Pax6; 1:500; $4^{\circ} \mathrm{C}$ for $24 \mathrm{~h}$; Developmental Studies Hybridoma Bank, Iowa, IA, USA); goat anti-sex determining region Y-box 2 (cat. no. AF2018; Sox 2; 1:500; $4^{\circ} \mathrm{C}$ for 24 h; R\&D Systems, Inc.); mouse anti-Tuj1 (cat. no. T8328; 1:500; $4^{\circ} \mathrm{C}$ overnight; Sigma-Aldrich, Merck KGaA); mouse anti-syntaxin 1 (cat. no. S0664; 1:1,000, $4^{\circ} \mathrm{C}$ overnight; Sigma-Aldrich, Merck KGaA); rabbit anti-calbindinD-28 K (cat.no. AB1778; $1: 500,4^{\circ} \mathrm{C}$ overnight; EMD Millipore); mouse anti-protein kinase $\mathrm{C} \alpha$ (cat.no. SAB4200739; PKC $\alpha, 1: 500,4^{\circ} \mathrm{C}$ overnight; Sigma-Aldrich, Merck KGaA); mouse anti-thy1.1 (cat. no. M7898; 1:100, $4^{\circ} \mathrm{C}$ overnight; Sigma-Aldrich, Merck $\mathrm{KGaA}$ ); rabbit anti-BrdU (cat. no. ab152095; $1: 200 ; 4^{\circ} \mathrm{C}$ overnight; Abcam); mouse anti-rhodopsin (cat. no. R5403; 1:2,000; $4^{\circ} \mathrm{C}$ overnight; Sigma-Aldrich, Merck KGaA); rabbit anti-phosphorylated (p)-histone H3 (cat. no. 9701S; pHH3; 1:1,000; $4^{\circ} \mathrm{C}$ overnight; Cell Signaling Technology, Inc.). Following extensive washing with PBS, cell coverslips or cryosections were treated with a 1:1,000 dilution of the following secondary antibodies from Invitrogen (Thermo Fisher Scientific, Inc.) for $1 \mathrm{~h}$ at room temperature in dark: Alexa Fluor 488-conjugated donkey anti-mouse immunoglobulin (IgG) (cat. no. A-21202); Alexa Fluor 488-conjugated donkey anti-rabbit IgG (cat. no. A-21206); Alexa Fluor 594-conjugated donkey anti-mouse IgG (cat. no. A-21203); Alexa Fluor 594-conjugated donkey anti-rabbit IgG (cat. no. A-21207); Alexa Fluor 594-conjugated donkey anti-goat IgG (cat. no. A-11058). DAPI was used for nuclear counterstaining (room temperature, $10 \mathrm{~min}$ ). The images of coverslips and sections were captured using fluorescence microscopy and confocal microscopy. Image J 1.50i software (National Institutes of Health, Bethesda, MD, USA) was used to determine the number of stained cells and the expression level of target proteins (measured by the integrated optical density and the relative IOD ratios) in stained sections. Cell counts were recorded from five coverslips in each culture condition, and at least three random fields were selected for cell counting. Five cryostat sections from each animal were selected for analysis, and at least three different animals were analyzed in each condition.
Semi-quantitative polymerase chain reaction (PCR) analysis. Following treatment, total RNA was isolated from cells using TRIzol reagent (Invitrogen; Thermo Fisher Scientific, Inc.), which was followed by cDNA synthesis with AMV reverse transcriptase and amplification with gene-specific forward and reverse primers (Table I) using a PCR kit (AMV) version 3.0 (Takara Biotechnology Co., Ltd., Dalian, China), as previously described (13). 30 cycles consisted of $30 \mathrm{sec}$ at $94^{\circ} \mathrm{C}, 30 \mathrm{sec}$ at $55^{\circ} \mathrm{C}$ and $1 \mathrm{~min}$ at $72^{\circ} \mathrm{C}$. PCR products were visualized on a $2 \%$ agarose gel. The images were detected with ethidium bromide staining using a Gel Doc ${ }^{\mathrm{TM}} \mathrm{XR}^{+}$system (Bio-Rad Laboratories, Inc., Hercules, CA, USA). GAPDH mRNA has been chosen as the internal standard in this study. Every experiment was repeated 3 times.

Western blot analysis. Following treatment, cell lysates were prepared for western blot analysis as previously described (13). Briefly, the cells were lysed on ice by sonication in radioimmunoprecipitation assay buffer (Beyotime Institute of Biotechnology, Haimen, China). The lysate was incubated on ice for $30 \mathrm{~min}$, centrifuged at a speed of $12,000 \mathrm{x} \mathrm{g}$ for $15 \mathrm{~min}$. Then, the supernatant was collected, protein concentrations were determined by the bicinchoninic acid method and $30 \mu \mathrm{g}$ protein of each sample was separated by $15 \%$ SDS-PAGE and transferred to $0.45 \mu \mathrm{m}$ polyvinylidene difluoride membranes (EMD Millipore). Membranes were blocked for $1 \mathrm{~h}$ at room temperature in 5\% non-fat dry milk in TBS with $0.01 \%$ Tween. The primary antibodies used for western blotting were as follows: Mouse anti-cyclinD1 (cat. no. 2926P; 1:1,000, $4^{\circ} \mathrm{C}$ overnight, Cell Signaling Technology, Inc.); mouse anti-cyclinD3 (cat. no. 2936S; 1:1,000, $4^{\circ} \mathrm{C}$ overnight, Cell Signaling Technology, Inc.); mouse anti-GAPDH (cat. no. KC-5G4; 1:10,000, $4^{\circ} \mathrm{C}$ overnight, KangChen Bio-tech, Inc., Shanghai, China). Horseradish peroxidase (HRP)-conjugated secondary antibodies were also used (cat. no. PA1-28761; 1:3,000, room temperature for $1 \mathrm{~h}$, Thermo Fisher Scientific, Inc.). Chemiluminescent immunoreactivity was executed by incubating the blot with the Immobilon Western HRP substrate (EMD Millipore) for $5 \mathrm{~min}$ at room temperature and detected using a ChemiDoc XRS+ System (Bio-Rad Laboratories, Inc.). 

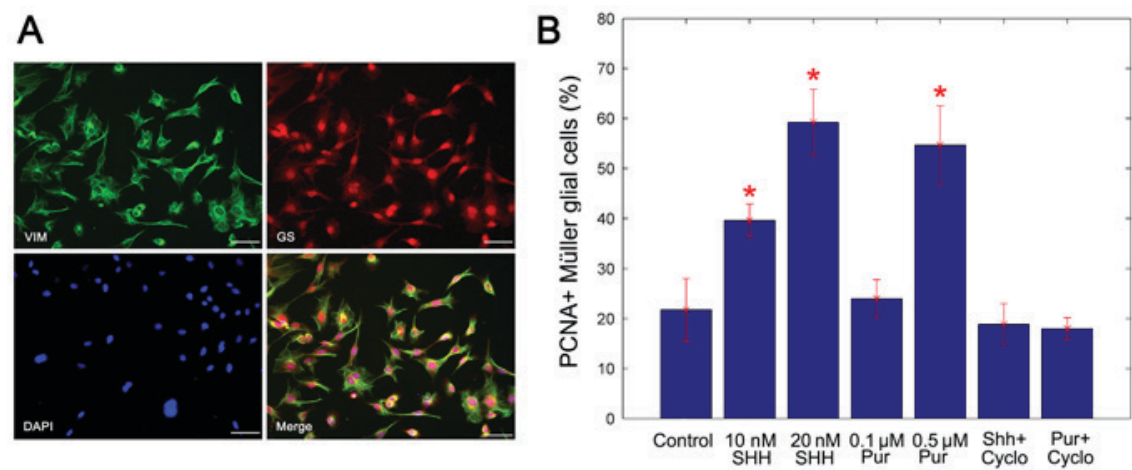

C
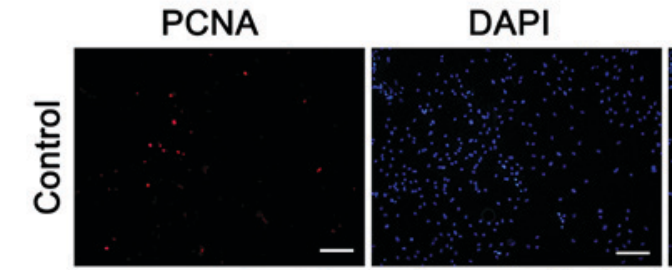

Merge
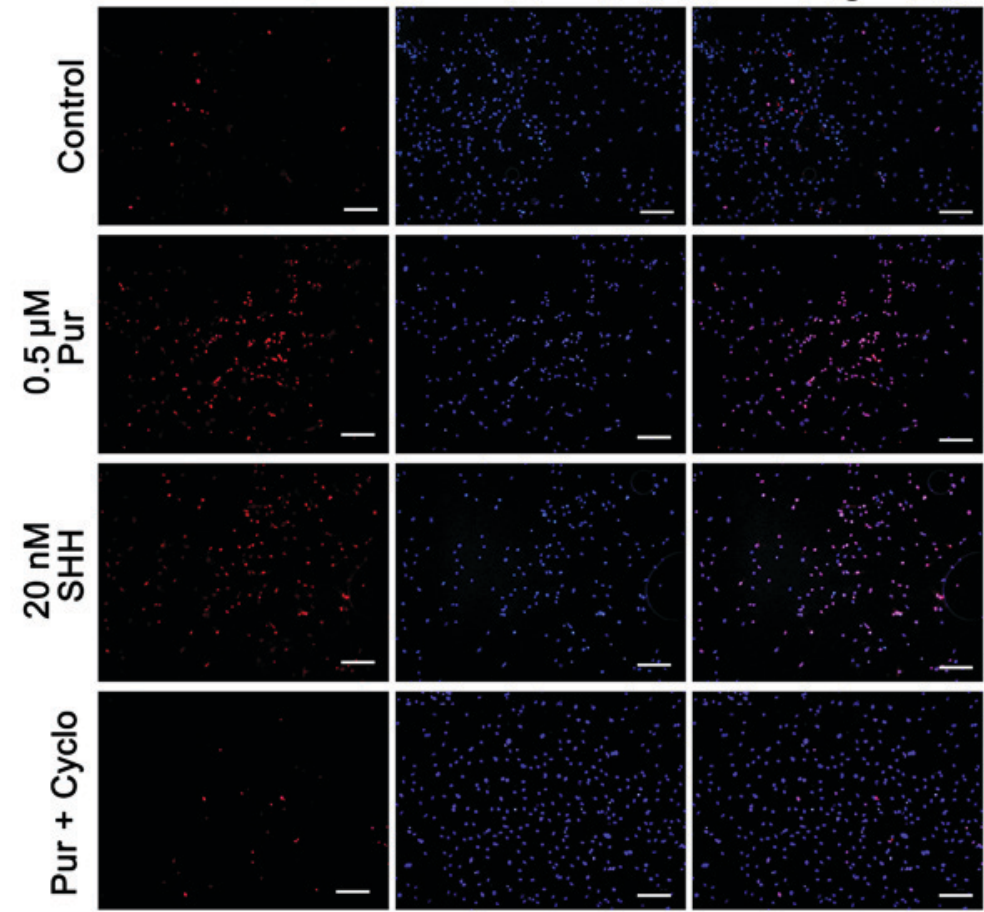

Figure 1. Purmorphamine promotes the proliferation of cultured Müller glial cells. (A) Double immunocytochemistry showed that cells co-expressed specific markers GS and Vimentin of Müller glial cells. Scale bar, $100 \mu \mathrm{m}$. (B) Percentage of PCNA+ Müller glial cells was carried by a statistical analysis. The mitogenic effect of $0.5 \mu \mathrm{M}$ purmorphamine was significantly greater than that the control and was similar to that of the $20 \mathrm{nM}$ SHH-N treatment. (C) Immunostaining was performed to analyze the percentage of PCNA-positive cells. When compared with the control, purmorphamine increased the percentage of PCNA-positive cells, as did treatment with SHH-N. However, co-treatment with purmorphamine and cyclopamine decreased the percentage of PCNA-positive cells. Scale bar, $200 \mu \mathrm{m} .{ }^{*} \mathrm{P}<0.05$ vs. control. Cyclo, cyclopamine; PCNA, proliferating cell nuclear antigen; Pur, purmorphamine; SHH, sonic hedgehog; GS, glutamine synthetase.

All the detections were repeated at least in 3 samples. Bands were analyzed using Gel-Pro Analyzer software (version 4.0, Media Cybernetics, Inc., MD, USA).

Statistical analysis. Differences between different groups were compared by one-way analysis of variance and Least Significant Difference post hoc analysis with Matlab R2012b (The Mathworks, Inc., MA, USA). Data were expressed as the mean \pm standard deviation. $\mathrm{P}<0.05$ was considered to indicate a statistically significant difference.

\section{Results}

Purmorphamine promotes the proliferation of Müller glial cells. Following 2 weeks of culture, immunocytochemistry revealed that cultured cells co-expressed the specific markers of Müller glia, GS and vimentin (Fig. 1A). The purity of cells achieved was $96 \%$ in fluorescence-activated cell sorting analysis as previously reported (14). The Müller glial cells were cultured for 2 days in the presence of different concentrations of SHH-N or purmorphamine, with or without cyclopamine. The immunocytochemistry analysis of PCNA was used to assay the proportion of cells in proliferation. Compared with the untreated control cells, the percentage of PCNA-positive Müller glia increased significantly following treatment with the two SHH-N concentrations and the $0.5 \mathrm{mM}$ purmorphamine treatment, whereas cyclopamine inhibited the production of PCNA-positive Müller glial cells compared with treatment with purmorphamine alone (representative immunostaining image are presented in Fig. 1B). In our previous study (14), the mitogenic effects of SHH was demonstrated to be concentration dependent, and no significant increase was identified at concentrations $>20 \mathrm{nM}$. In the present study, the mitogenic effect of $0.5 \mu \mathrm{M}$ purmorphamine was similar to that of $20 \mathrm{nM}$ 

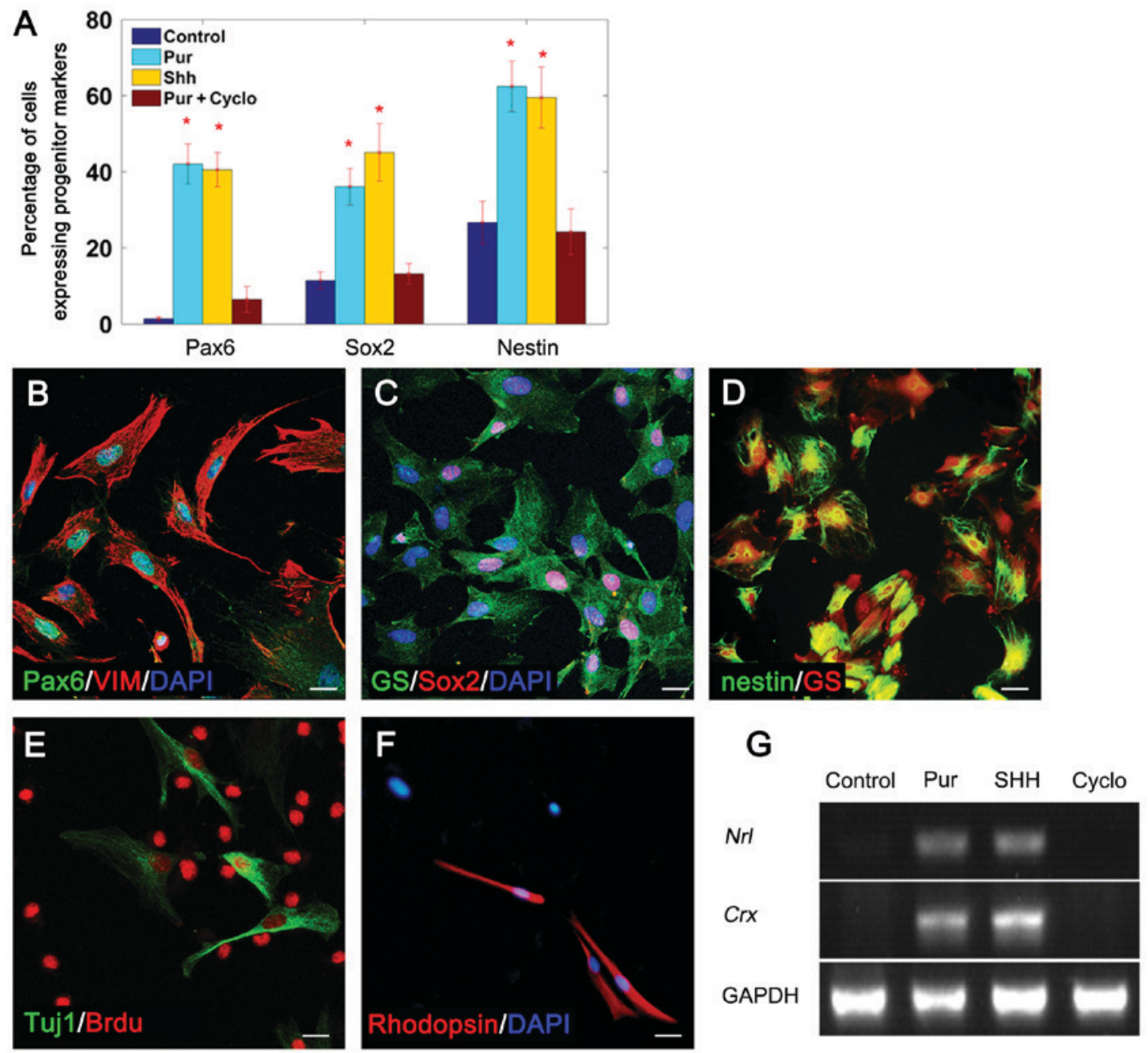

Figure 2. Müller glial cells dedifferentiated into progenitor cells and generated rod-like cells in the presence of purmorphamine. (A) The percentage of cells expressing progenitor cell markers under different treatment conditions. Double staining revealed that purmorphamine treatment induced Müller glia (VIM- or GS-positive) to express the progenitor cell markers (B) Pax6, (C) Sox2 and (D) nestin. Following 2 days in fresh medium culture, Müller glia-derived progenitor cells expressed (E) the neuron marker Tuj1 and (F) the rod cell-specific marker rhodopsin. (G) mRNA transcripts of rod development genes, $n r l$ and $\operatorname{cr} x$, were detected by semi-quantitative polymerase chain reaction following treatment with purmorphamine or SHH-N; however, no expression was detected in control cells or cells co-treated with cyclopamine. "P<0.05 vs. control. Scale bars, $30 \mu \mathrm{m}$. SHH+cyclo, data not shown. BrdU, 5-bromo-2'-deoxy uridine; cyclo, cyclopamine; crx, cone-rod homeobox; GS, glutamine synthetase; $n r l$, neural retina-specific leucine zipper; Pax6, paired box protein-6; Pur, purmorphamine; SHH, sonic hedgehog; Sox2, sex determining region Y-box 2; Tuj1, specific class III $\beta$-tubulin; VIM, vimentin.

SHH-N (Fig. 1B and C). Therefore, $0.5 \mu \mathrm{M}$ purmorphamine and $20 \mathrm{~nm}$ SHH-N were used in subsequent experiments. These data indicated that the proliferation of Müller glial cells was promoted by purmorphamine treatment as well as SHH-N.

Purmorphamine-treated Müller glial cells express progenitor cell markers. To determine whether purmorphamine treatment stimulated Müller glial cells to dedifferentiate into progenitor-like cells, Müller glial cells were cultured in the presence of $20 \mathrm{nM}$ SHH-N or $0.5 \mathrm{mM}$ purmorphamine for 7 days, with or without cyclopamine. Purmorphamine treatment induced the expression of the retinal progenitor cell marker Pax6 in $42.05 \pm 5.25 \%$ of cells (Fig. $2 \mathrm{~A}$ and B), the neurogenic gene Sox 2 in $36.08 \pm 4.8 \%$ of cells (Fig. $2 \mathrm{~A}$ and C) and the neural precursor marker nestin in $62.44 \pm 6.63 \%$ of cells (Fig. $2 \mathrm{~A}$ and $\mathrm{D}$ ). In addition, the effects of purmorphamine were similar to those of SHH-N (Fig. 2A). The expression of these progenitor cell markers was markedly reduced when cells were co-treated with purmorphamine and cyclopamine (Fig. 2A) (SHH with cyclopamine, data not shown). These results suggested that purmorphamine may stimulate the multi-potency of Müller glia.

Müller glia-derived cells express Tuj1 and rhodopsin following purmorphamine treatment. The regenerative potential of purmorphamine-treated Müller glia was further investigated. Cells were cultured in purmorphamine with or without cyclopamine for 7 days, and then grown in fresh medium without purmorphamine for a further 2 days. Following this, cells were collected for immunofluorescence staining and PCR analysis. Immunofluorescence indicated that Müller glia-derived cells expressed the neuronal marker Tuj-1 (Fig. 2E) and the rod cell-specific marker rhodopsin (Fig. 2F), which suggested that they have altered their neuronal lineage and have obtained the ability to generate retinal neurons. The PCR results demonstrated that purmorphamine induced Müller glial cells to express neural retina-specific leucine zipper $(n r l)$ and cone-rod homeobox ( $c r x$; Fig. 2G), which have been reported to participate in the generation of rod cells and the regulation of rhodopsin transcription (26). To examine whether 
Müller glia-derived cells differentiated into other retinal cell types, specific antibodies for retinal neurons ( $\mathrm{PKC} \alpha$ for bipolar, calbindin for horizontal, syntaxin1 for amacrine and Thy1.1 for ganglion cells) were used; however, expression of these markers was not detected. These data revealed that purmorphamine treatment promoted Müller glial cells to transdifferentiate in the direction of retinal neurons.

Purmorphamine promotes retinal regeneration following photoreceptor degeneration. As aforementioned, purmorphamine stimulated the proliferation and transdifferentiation of Müller glial cells in vitro in a similar manner as SHH-N treatment. The present study subsequently evaluated whether purmorphamine treatment was able to control the proliferative and regenerative competence of these cells in vivo. Intraocular injections of purmorphamine or SHH-N with or without cyclopamine were administered to MNU-exposed rats; the control retinas were treated with physiological saline. The proliferative ability of cells was assessed by $\mathrm{pHH} 3$ and vimentin double-immunofluorescence staining on day 3 (Fig. 3A-D). The results demonstrated that MNU-induced degeneration of photoreceptors stimulated Müller glia re-entry into the cell cycle in control retina, and there were $62.33 \pm 6.67 \mathrm{pHH}^{+}$cells/section distributed in the inner nuclear layer, 3 days following the injection of MNU (Fig. 3A and E). Treatment with purmorphamine or SHH-N for 3 days, increased the number of proliferating cells detected, with 106.22 \pm 7.22 pHH3 $^{+}$cells/section (Fig. 3B and E) and $101.44 \pm 11.91 \mathrm{pHH} 3^{+}$cells/section (Fig. 3C and E), respectively. However, proliferation decreased to only $28.78 \pm 7.63$ $\mathrm{pHH}^{+}$cells/section in retinas co-treated with purmorphamine and cyclopamine (Fig. 3D and E) (SHH + cyclopamine, data not shown).

To verify the capacity of Müller glial cells to generate neurons, the expression of progenitor cell markers nestin and Pax6 were detected on day 3 (Fig. 3F-J and K-O, respectively). The expression of nestin increased following purmorphamine treatment (Fig. 3G and J) as observed via IOD analysis. The IOD ratio of nestin/GS was $37.23 \pm 1.80 \%$ in purmorphamine-treated retina (Fig. $3 \mathrm{G}$ and $\mathrm{J}$ ) and $10.22 \pm 1.15 \%$ in the control retina (Fig. 3F and J). Following retinal injury, $39.67 \pm 4.56$ cells/section were double positive for Pax6 and vimentin in the control group (Fig. $3 \mathrm{~K}$ and $\mathrm{O}$ ). This population increased following the administration of purmorphamine or SHH-N, with $87.89 \pm 11.82$ cells/section (Fig. 3L and O) and $92.22 \pm 11.54$ cells/section (Fig. $3 \mathrm{M}$ and O), respectively. It was concluded that purmorphamine was able to stimulate the proliferation of Müller glia-derived progenitors following retinal injury similar to SHH.

To observe the effects of purmorphamine on the fate of Müller glia-derived progenitors, rats were injected daily for 7 consecutive days immediately following the application of MNU, and were sacrificed on day 1,7 and 15. Compared with the retina on day 1 (Fig. 3P and Q), rhodopsin-positive cells were not detected when the ONL disappeared on day 7 following MNU-treatment (Fig. 3R and S). However, rhodopsin-positive rod-like cells reappeared in purmorphamine-treated retina on day 15 compared to control retina (Fig. 3T and U).

Proliferation of Müller glial cells is induced by purmorphamine through cyclin D1 and cyclin D3. It has been previously reported that the proliferation of Müller glia is induced via the cyclin D1 and D3 associated pathway, and that SHH promoted this effect (13). To determine whether the effects of purmorphamine are implemented through cyclin D1 and cyclin D3, western blotting was performed 3 days following the addition of purmorphamine or SHH-N, to evaluate the expression of cyclin D1 and D3. The results revealed that the expression of these two proteins was increased in purmorphamine and SHH-stimulated Müller glial cells (Fig. 4A). Semi-quantitative PCR analysis revealed that cultured Müller glial cells expressed the SHH target genes smoothened homolog precursor (smo) and GLI family zinc-finger 1 ( $g l i 1$ ), and the levels of these mRNA transcripts increased with the addition of purmorphamine and decreased in cells co-treated with cyclopamine (Fig. 4B) (SHH + cyclopamine, data not shown). It was concluded that purmorphamine promotes the proliferation of Müller glial cells by activating the SHH-pathway. Cyclin D1 and cyclin D3 expression levels have been demonstrated to be increased by Wnt signaling pathway activation (16), and $\mathrm{SHH}$ activates the Wnt signaling pathway by regulating the expression of E-cadherin $(27,28)$. Thus, the Wnt signaling pathway inhibitor, DKK1, was added to purmorphamine-stimulated Müller glial cells. The proliferative ability of Müller glial cells was not affected by the presence of DKK1 (Fig. 4C and D). The data indicated that purmorphamine preferentially induced the expression of cyclin D1 and cyclin D3 through the activation of smo and glil, which in turn promoted the proliferation of Müller glial cells.

\section{Discussion}

In the past decade, there has been a paradigm shift in our understanding of glial cells during the development and regeneration of central nervous system (CNS). Glial cells provide homeostatic support and serve as a source of stem cells in the embryonic brain and the adult subventricular zone (SVZ) and subgranular layer (SGL) $(5,12)$; however, active neurogenesis has not been detected in the adult mammalian retina (7). By contrast, neural regeneration has been observed in the injured retina, and the source of injury-induced neurogenesis has been traced to Müller glia $(8,15,29)$. In addition, the application of SHH was able to facilitate the process $(13,14,30,31)$. The results of the present study demonstrated that the application of purmorphamine, an agonist of the SHH signaling pathway, promoted Müller glia proliferation, dedifferentiation into progenitor cells and the production of retinal neurons in vitro. The proliferating cells co-expressed GS/vimentin and various progenitor cell markers, including Pax6, Sox 2 and nestin, alluding to their progenitor nature. Intraocular injections of purmorphamine also promoted retinal regeneration through Müller glia as demonstrated by the increased number of proliferating Müller glial cells and the re-expression of rhodopsin.

The response of Müller glia to disease and injury includes the upregulated expression of glial fibrillary acidic protein, an intermediate filament protein, cell hypertrophy and proliferation, which is common to glia in the CNS, collectively known as reactive gliosis (32). Not all injuries and diseases lead to the proliferation of retinal Müller glia. Rapid retinal degeneration typically leads to Müller glia proliferation; however, in slow progressing retinal degeneration, such as that exhibited by 

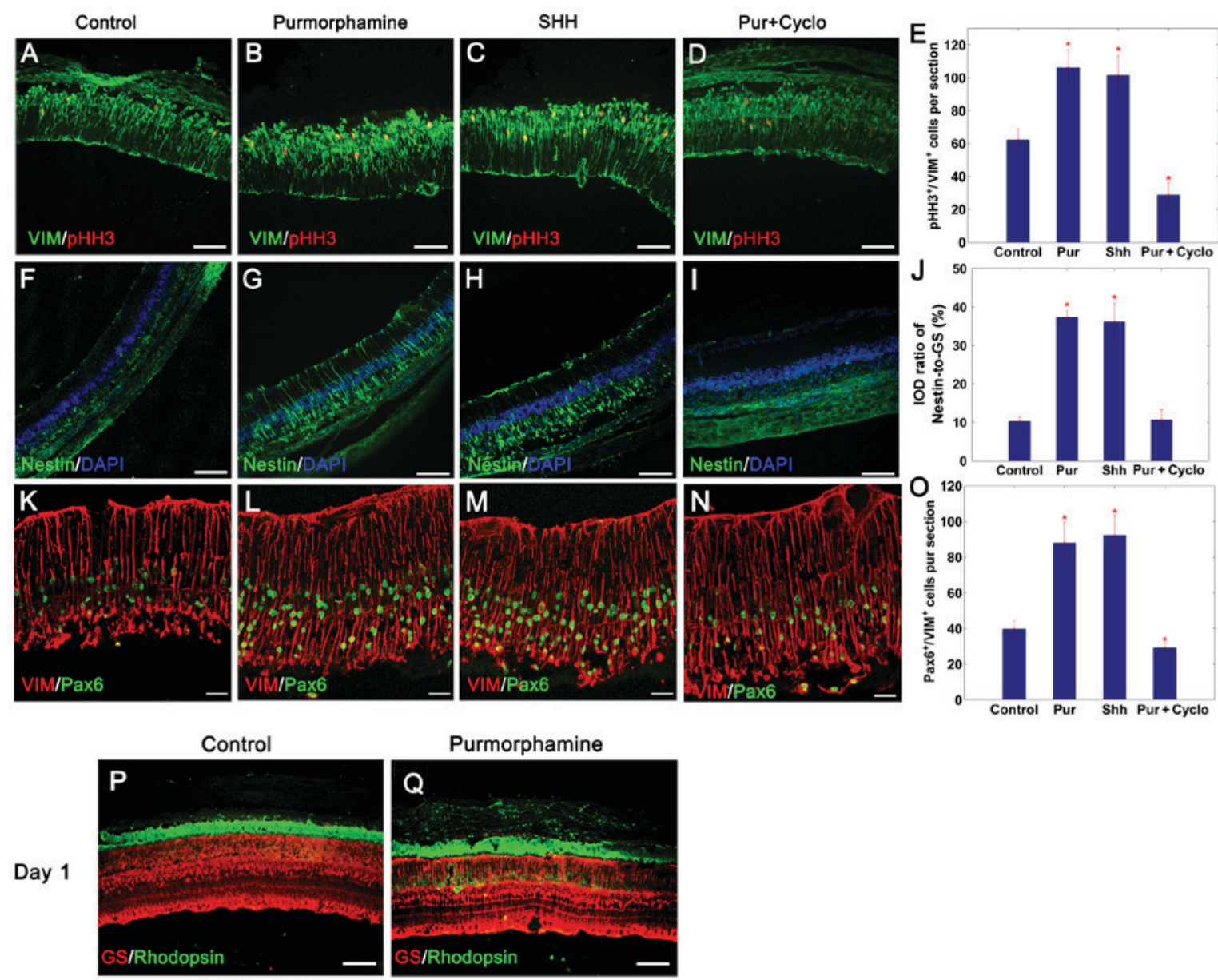

Purmorphamine
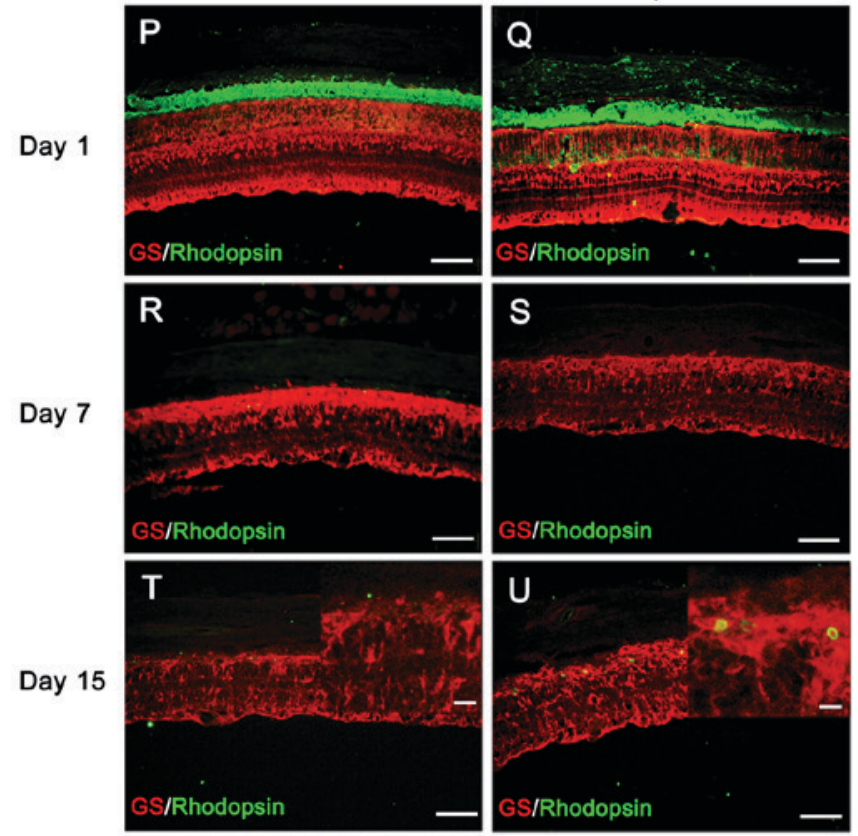

Figure 3. Purmorphamine promotes retinal regeneration in photoreceptor damaged retina. Compared with the (A) control, the percentage of $\mathrm{pHH} 3^{+} / \mathrm{VIM}^{+} \mathrm{Müller}$ glia increased significantly following an intravitreal injection of (B) purmorphamine or (C) SHH-N on day 3. This mitogenic effect was inhibited in purmorphamine + cyclopamine treated Müller glial cells (D) SHH + cyclopamine, data not shown. (E) pHH3-labeling images were analyzed to identify the number of pHH3/vimentin-positive cells. (F-I) Following retinal damage, Müller glia dedifferentiated into progenitor cells by expressing nestin. (J) The IOD ratio of nestin/GS in purmorphamine-treated retina was significantly higher than that exhibited by control retina (images not available, only the graph is displayed). (K-N) Following retinal damage, Müller glia dedifferentiated into progenitor cells by expressing Pax6. (O) In addition, the number of Pax6 $6^{+} \mathrm{VIM}^{+}$cells was significantly greater compared with the control; the levels were also similar to those produced by SHH treatment. (P and Q) Photoreceptor degeneration was induced by MNU injection. With the progression of photoreceptor degeneration, the expression of rhodopsin completely disappeared on day 7 (R and S), with and without purmorphamine. However, there were more rhodopsin-positive cells produced with the purmorphamine injection, when compared with the control retina on day 15 (T and U). "P<0.05 vs. control. Scale bars: A-D, F-I and P-S, $100 \mu \mathrm{m}$; K-N, $30 \mu \mathrm{m}$; the magnified images in (T) and (U), $10 \mu \mathrm{m}$. Cyclo, cyclopamine; GS, glutamine synthetase; IOD, integrated optical density; Pax6, paired box protein-6; pHH3, phosphorylated-histone H3; Pur, purmorphamine; SHH, sonic hedgehog; VIM, vimentin.

Borna disease virus-infected and Royal College of Surgeons (RCS) rats, retinal gliosis continues to be nonproliferative $(32,33)$. Given that the initial stages of reactive gliosis have been observed to be neuroprotective and regenerative (32), extended and excessive proliferation may serve as a barrier towards regeneration just as it does in the CNS. The rapid downregulation of cyclin D1 and cyclin D3 expression was previously reported to prevent uncontrolled proliferation of Müller glia, and resulted in cells exiting mitosis and initiating differentiation (34). In the present study, there was $\sim 2.5$ times 


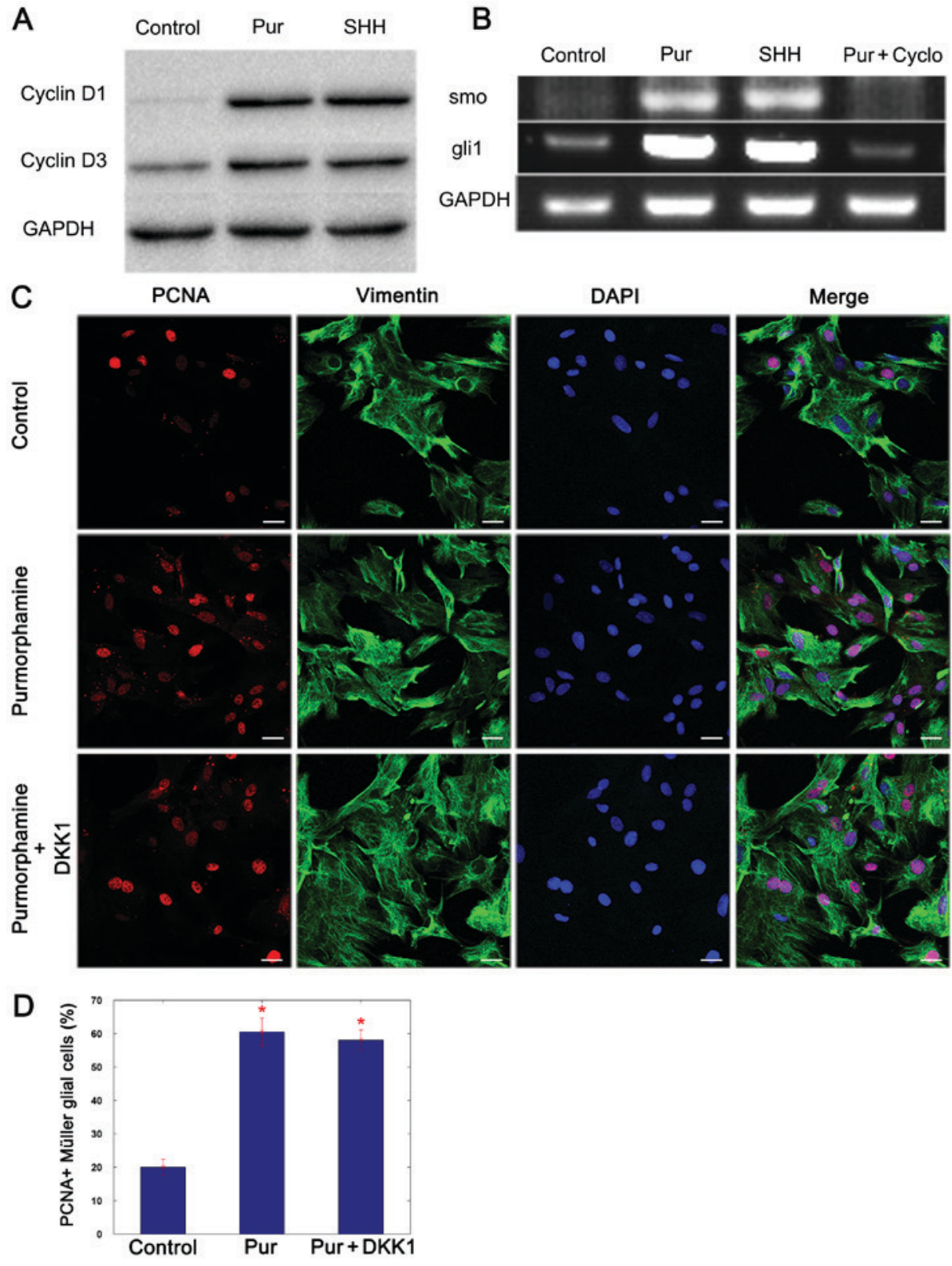

Figure 4. Purmorphamine increases the expression of cyclin D1 and cyclin D3 proteins by preferentially activating the SHH signaling pathway. (A) Western blot analysis revealed that the protein expression levels of cyclin D1 and cyclin D3 were increased 3 days following treatment with purmorphamine or SHH-N compared with control. (B) Semi-quantitative polymerase chain reaction demonstrated that the expression of smo and gli1 mRNA increased when cells were treated with purmorphamine or SHH-N; however, the level of the transcripts decreased in the presence of cyclopamine. (C and D) Double staining of PCNA and vimentin indicated that the proliferation of Müller glial cells was induced by purmorphamine; however, it was not affected by treatment with the Wnt signaling pathway inhibitor DKK1. "P<0.05 vs. control. Scale bars, $30 \mu \mathrm{m}$. Cyclo, cyclopamine; DKK1, Dickkopf-related 1; gli1, GLI family zinc-finger 1; PCNA, proliferating cell nuclear antigen; Pur, purmorphamine; SHH, sonic hedgehog; smo, smoothened homolog precursor.

increase in mitotic Müller glial cells which were induced by purmorphamine, when compared with the control (Fig. 1B). However, the period of proliferation was not prolonged, and the number of dividing cells reached a peak on day 3 , which then decreased on day 7 in the same manner as the controls (data not shown). These results suggested that the application of purmorphamine may not cause excessive proliferation, thus, the formation of glial scar tissue may be avoided.

The importance of SHH has been demonstrated in the maintenance of stem cells and progenitors in the CNS (35). SHH was reported to stimulate the formation of proliferating Müller glia-derived progenitor cells in chick and rat retina $(13,14,36)$. However, owing to the variable activity and the high cost of megadose utilization, particularly for long-term treatment, the application of recombinant SHH may be limited. Cell-permeable small molecules may be a potential solution as they have similar biological functions, stable activity and are available at a low price (37). One such molecule is purmorphamine, which has been demonstrated previously to activate glil expression, a downstream target of the SHH signaling pathway $(22,38)$. The present study demonstrated that purmorphamine promoted the proliferation and dedifferentiation of Müller glia, and facilitated its fate shift to a neuronal lineage, in the same manner as SHH treatment does. The use of purmorphamine achieves efficient transdifferentiation, decreases the cost and also makes in situ 
regeneration feasible as it has a stable chemical nature and has an easy preparation procedure.

Extrinsic and intrinsic factors participate in the activation process associated with Müller glial cells in retinal regeneration, including $\mathrm{SHH}(14)$, Notch $(15)$, Wnt $(16,17)$ and fibroblast growth factor (18) signaling pathways. Activation of these pathways, due to injury or exogenous ligands, induces a subset of Müller glial cells into the G1-S phase of the cell cycle $(6,39)$. Our previous study indicated that SHH may promote the proliferation of Müller glial cells by increasing the expression of cyclin D1 and cyclin D3 (14). It has been reported that cyclin D1 expression may be increased by Wnt signaling pathway activation (16); $\mathrm{SHH}$ is also a crucial signaling pathway, which mostly functions with other morphogens, Wnts in particular, rather than alone (40-42). Therefore, in the present study, the Wnt signaling pathway inhibitor DKK1 was applied to the culture medium. Purmorphamine treatment activated the SHH signaling pathway, and increased the levels of mRNA for glil and smo, and the protein expression of cyclin D1 and cyclin D3, even in the presence of DKK1. As DKK1 was an inhibitor of the Wnt signaling pathway, these results suggested that purmorphamine may not function through the Wnt pathway but induce the proliferation of Müller glial cells by directly upregulating the expression of cyclin D1 and cyclin D3 which is similar to SHH. However, there is the possibility that there may be other unknown pathways involved in this process, thus, further research is required.

In conclusion, purmorphamine treatment efficiently promoted the proliferation of Müller glial cells and the production of photoreceptors, as an agonist of SHH. These results provide evidence to support the possibility that small molecules may be used as an alternative treatment strategy for the endogenous regeneration of the retina via Müller glial cells. Further investigations are required to focus on the efficient generation of specific retinal neurons from Müller glia-derived cells to enhance the potential of repair in the injured retina.

\section{Acknowledgements}

The present study was supported by The National Natural Science Foundation of China (grant nos. 30971536 and 31571238).

\section{References}

1. Fischer AJ and Reh TA: Müller glia are a potential source of neural regeneration in the postnatal chicken retina. Nat Neurosci 4: 247-252, 2001.

2. Hamon A, Roger JE, Yang XJ and Perron M: Müller glial cell-dependent regeneration of the neural retina: An overview across vertebrate model systems. Dev Dyn 245: 727-738, 2016.

3. Brockerhoff SE and Fadool JM: Genetics of photoreceptor degeneration and regeneration in zebrafish. Cell Mol Life Sci 68: 651-659, 2011

4. Wang SZ and Yan RT: The retinal pigment epithelium: A convenient source of new photoreceptor cells? J Ophthalmic Vis Res 9: 83-93, 2014.

5. Kriegstein A and Alvarez-Buylla A: The glial nature of embryonic and adult neural stem cells. Annu Rev Neurosci 32: 149-184, 2009.

6. Wilken MS and Reh TA: Retinal regeneration in birds and mice. Curr Opin Genet Dev 40: 57-64, 2016.

7. Moshiri A and Reh TA: Persistent progenitors at the retinal margin of $\mathrm{ptc}^{+/-}$mice. J Neurosci 24: 229-237, 2004.
8. Karl MO, Hayes S, Nelson BR, Tan K, Buckingham B and Reh TA: Stimulation of neural regeneration in the mouse retina. Proc Natl Acad Sci USA 105: 19508-19513, 2008.

9. Goldman D: Müller glial cell reprogramming and retina regeneration. Nat Rev Neurosci 15: 431-442, 2014.

10. Lenkowski JR and Raymond PA: Muller glia: Stem cells for generation and regeneration of retinal neurons in teleost fish. Prog Retin Eye Res 40: 94-123, 2014.

11. Raymond PA, Barthel LK, Bernardos RL and Perkowski JJ: Molecular characterization of retinal stem cells and their niches in adult zebrafish. BMC Dev Biol 6: 36, 2006.

12. Ahmad I, Del Debbio CB, Das AV and Parameswaran S: Müller glia: A promising target for therapeutic regeneration. Invest Ophthalmol Vis Sci 52: 5758-5764, 2011.

13. Wan J, Zheng H, Chen ZL, Xiao HL, Shen ZJ and Zhou GM: Preferential regeneration of photoreceptor from Müller glia after retinal degeneration in adult rat. Vision Res 48: 223-234, 2008.

14. Wan J, Zheng H, Xiao HL, She ZJ and Zhou GM: Sonic hedgehog promotes stem-cell potential of Müller glia in the mammalian retina. Biochem Biophys Res Commun 363: 347-354, 2007.

15. Del Debbio CB, Balasubramanian S, Parameswaran S, Chaudhuri A, Qiu F and Ahmad I: Notch and Wnt signaling mediated rod photoreceptor regeneration by Müller cells in adult mammalian retina. PLoS One 5: e12425, 2010.

16. Osakada F, Ooto S, Akagi T, Mandai M, Akaike A and Takahashi M: Wnt signaling promotes regeneration in the retina of adult mammals. J Neurosci 27: 4210-4219, 2007.

17. Liu B, Hunter DJ, Rooker S, Chan A, Paulus YM, Leucht P, Nusse Y, Nomoto $\mathrm{H}$ and Helms JA: Wnt signaling promotes Müller cell proliferation and survival after injury. Invest Ophthalmol Vis Sci 54: 444-453, 2013.

18. Fischer AJ and Bongini R: Turning Müller glia into neural progenitors in the retina. Mol Neurobiol 42: 199-209, 2010.

19. Stenkamp DL, Frey RA, Prabhudesai SN and Raymond PA: Function for hedgehog genes in zebrafish retinal development. Dev Biol 220: 238-252, 2000

20. Moshiri A, McGuire CR and Reh TA: Sonic hedgehog regulates proliferation of the retinal ciliary marginal zone in posthatch chicks. Dev Dyn 233: 66-75, 2005.

21. Jensen AM and Wallace VA: Expression of sonic hedgehog and its putative role as a precursor cell mitogen in the developing mouse retina. Development 124: 363-371, 1997.

22. Sinha S and Chen JK: Purmorphamine activates the Hedgehog pathway by targeting Smoothened. Nat Chem Biol 2: 29-30, 2006.

23. ARVO: Toolkit for Biomedical Researchers Using Laboratory Animals. http://www.arvo.org/Journals and_Publications/ Toolkit_for_Biomedical_Researchers_Using_Laboratory_Animals. Accessed Nov 17, 2014

24. Shanghai Experimental Animal Management Method. http://www.

shanghai.gov cn/nw2/nw2314/nw2319/nw2407/nw26170/u26aw27198.html.

25. Fudan University Guide for the Care and Use of Laboratory Animals. http://lsem.fudan.edu.cn/wz/websit/article.jsp.

26. Ohsawa R and Kageyama R: Regulation of retinal cell fate specification by multiple transcription factors. Brain Res 1192: 90-98, 2008.

27. Xiao C, Ogle SA, Schumacher MA, Orr-Asman MA, Miller ML, Lertkowit N, Varro A, Hollande F and Zavros Y: Loss of parietal cell expression of Sonic hedgehog induces hyperproliferation of surface mucous cells. Gastroenterology 138: 550-561, 561.e1-e8, 2010.

28. Xiao C, Ogle SA, Schumacher MA, Schilling N, Tokhunts RA, Orr-Asman MA, Miller ML, Robbins DJ, Hollande F and Zavros Y: Hedgehog signaling regulates E-cadherin expression for the maintenance of the actin cytoskeleton and tight junctions. Am J Physiol Gastrointest Liver Physiol 299: G1252-G1265, 2010.

29. Ooto S, Akagi T, Kageyama R, Akita J, Mandai M, Honda Y and Takahashi M: Potential for neural regeneration after neurotoxic injury in the adult mammalian retina. Proc Natl Acad Sci USA 101: 13654-13659, 2004.

30. Todd L and Fischer AJ: Hedgehog signaling stimulates the formation of proliferating Muller glia-derived progenitor cells in the chick retina. Development 142: 2610-2622, 2015.

31. Ferraro S, Gomez-Montalvo AI, Olmos R, Ramirez M and Lamas M: Primary ciliain rat mature Muller glia: Downregulation of IFT20 expression reduces sonic hedgehog-mediated proliferation and dedifferentiation potential of Muller glia primary cultures. Cell Mol Neurobiol 35: 533-542, 2015. 
32. Bringmann A, Iandiev I, Pannicke T, Wurm A, Hollborn M, Wiedemann P, Osborne NN and Reichenbach A: Cellular signaling and factors involved in Müller cell gliosis: Neuroprotective and detrimental effects. Prog Retin Eye Res 28: 423-451, 2009.

33. Iandiev I, Biedermann B, Bringmann A, Reichel MB, Reichenbach A and Pannicke T: Atypical gliosis in Müller cells of the slowly degenerating rds mutant mouse retina. Exp Eye Res 82: 449-457, 2006.

34. Dyer MA and Cepko CL: Control of Muller glial cell proliferation and activation following retinal injury. Nat Neurosci 3 . $873-880,2000$

35. Fuccillo M, Joyner AL and Fishell G: Morphogen to mitogen: The multiple roles of hedgehog signalling in vertebrate neural development. Nat Rev Neurosci 7: 772-783, 2006.

36. Todd L and Fischer AJ: Hedgehog signaling stimulates the formation of proliferating Müller glia-derived progenitor cells in the chick retina. Development 142: 2610-2622, 2015.

37. Ding S and Schultz PG: A role for chemistry in stem cell biology. Nat Biotechnol 22: 833-840, 2004.
38. Chechneva OV, Mayrhofer F, Daugherty DJ, Krishnamurty RG, Bannerman P, Pleasure DE and Deng W: A Smoothened receptor agonist is neuroprotective and promotes regeneration after ischemic brain injury. Cell Death Dis 5: e1481, 2014.

39. Xia X and Ahmad I: Unlocking the neurogenic potential of mammalian Muller glia. Int J Stem Cells 9: 169-175, 2016.

40. Aviles EC, Wilson NH and Stoeckli ET: Sonic hedgehog and Wnt: Antagonists in morphogenesis but collaborators in axon guidance. Front Cell Neurosci 7: 86, 2013.

41. Wilson NH and Stoeckli ET: Sonic Hedgehog regulates Wnt activity during neural circuit formation. Vitam Horm 88: 173-209, 2012.

42. Tang M, Villaescusa JC, Luo SX, Guitarte C, Lei S, Miyamoto Y, Taketo MM, Arenas E and Huang EJ: Interactions of Wnt/ beta-catenin signaling and sonic hedgehog regulate the neurogenesis of ventral midbrain dopamine neurons. J Neurosci 30: 9280-9291, 2010 